\title{
Evolution of noble gas and water isotopes along the regional groundwater flow path of the Konya Closed Basin, Turkey
}

\author{
N. Nur Ozyurt* and C. Serdar Bayari \\ Geological Engineering Department, Hacettepe University, Beytepe 06800 Ankara, Turkey
}

\begin{abstract}
Noble gas and water isotope compositions of regional groundwater were investigated along two transects in the Konya Closed Basin (KCB) of central Turkey. According to the ${ }^{3} \mathrm{He} /{ }^{4} \mathrm{He}$ versus $\mathrm{Ne} / \mathrm{He}$ plot of samples, crust (up to $86 \%$ ) and mantle (up to $26 \%$ ) appear to be the primary and secondary sources of dissolved $\mathrm{He}$ in groundwater, respectively. After the beginning of both transects where the flow domain is confined, both ${ }^{3} \mathrm{He}$ and ${ }^{4} \mathrm{He}$ accumulate steadily in groundwater. Thereafter, the intermediate recharge from the surface in the unconfined part of regional flow system disrupts the steady accumulation trend of ${ }^{3} \mathrm{He}$ and ${ }^{4} \mathrm{He}$. Effect of intermediate recharge on regional groundwater is also indicated by the spatial variations in specific conductance, temperature, water isotopes, and tritium signals. At the last part of the flow domain where the system becomes confined again ${ }^{3} \mathrm{He}$ and ${ }^{4} \mathrm{He}$ start to accumulate in the eastern transect whereas they continue to decline in the western transect probably because of degassing to atmosphere through thinner confining unit. The ${ }^{4} \mathrm{He}$ accumulation rates based on radiocarbon ages and ${ }^{4} \mathrm{He}$ concentrations along both transects between the mountain flank and Obruk Plateau are in agreement with literature values but differ from each other. The accumulation rate along the eastern and western transects are $4.93 \times 10^{-11} \mathrm{~cm}^{3}$ STP/g•year and $1.99 \times 10^{-10} \mathrm{~cm}^{3} \mathrm{STP} / \mathrm{g} \bullet$ year, respectively. These values reveal that the ${ }^{4} \mathrm{He}$ accumulation rates may differ at least four times at different parts of a single aquifer, which are located 30 to $60 \mathrm{~km}$ apart. It is understood that any groundwater age-dating attempt by ${ }^{4} \mathrm{He}$ requires a firm pre-assessment of spatial ${ }^{4} \mathrm{He}$ accumulation rates. He isotope signal in the groundwater of KCB proves the mantle and/or crustal gas input into the carbonate aquifer in which an on-going hypogenic karst development has been suspected.
\end{abstract}

Keywords: $\quad$ noble gas, water isotopes, Konya Closed Basin, Turkey

Received 13 January 2018; Revised 8 November 2018; Accepted 8 November 2018

Citation: Ozyurt N.N. and Bayari C.S., 2018. Evolution of noble gas and water isotopes along the regional groundwater flow path of the Konya Closed Basin, Turkey. International Journal of Speleology, 47 (3), 333-342. Tampa, FL (USA) ISSN 0392-6672 https://doi.org/10.5038/1827-806X.47.3.2176

\section{INTRODUCTION}

Noble gases ( $\mathrm{He}, \mathrm{Ne}, \mathrm{Ar}, \mathrm{Kr}, \mathrm{Xe}$, and $\mathrm{Rn}$ ) are released into the environment mainly from atmosphere, mantle and crust. Since noble gases are not involved commonly in chemical reactions, their isotopic signals can be used to identify various natural processes (e.g., Rozanski, 1992; Solomon, 2000). The use of noble gases in geochemistry started in early 1900s, mostly for geochronological applications (e.g., Ozima \& Podosek, 1983). Later on, in 1970s, following the establishment of data on their solubility in water, they have been used in hydrogeological and hydrogeochemical studies. Today, noble gases (e.g., $\mathrm{He}, \mathrm{Ne}, \mathrm{Ar}, \mathrm{Kr}$, and $\mathrm{Xe}$ ) are widely used for estimating the recharge temperature, dating (based on tritiumtritiogenic helium-3 ratios) and characterization of the groundwater flow in large aquifer systems. Among all noble gases, helium isotopes (i.e., ${ }^{3} \mathrm{He}$ and ${ }^{4} \mathrm{He}$ ) are particularly useful in geological and hydrogeological applications because of their characteristic endmember signals of the sources of atmosphere, crust and mantle (e.g., Solomon, 2000). Many researchers attempted to use He for determining the groundwater travel times (e.g., Andrews \& Lee, 1979; Torgersen, 1980; Andrews et al., 1982, 1985; Torgersen \& Ivey, 1985; Balderer \& Lehmann, 1989; Mazor \& Bosch, 1991, 1992; Bottomley et al., 1990; Fu et al., 1990; Ballentine et al., 1991; Stute et al., 1992; Marty et al., 1993). Observations on groundwater's helium concentration along the regional flow path in the Bunter Sandstone, England (Andrews \& Lee, 1979) and in the Great Artesian Basin, Australia (Torgersen \& Clarke, 1985) showed good correlation between the groundwater's $\mathrm{He}$ content and travel time. However, as Solomon (2000) indicates, "Although the 
concentration of ${ }^{4} \mathrm{He}$ and ${ }^{14} \mathrm{C} /$ hydraulic ages are well correlated, it has also been observed that the $\mathrm{He}$ accumulation rate is generally significantly larger than what can be explained by the release from $U$ and $T h$ decay within the aquifer. The excess $\mathrm{He}$ is attributed to input from crustal rocks and from the mantle (e.g., Andrews et al., 1982; Torgersen \& Clarke, 1985; Torgersen \& Ivey, 1985; Stute et al., 1992; Marty et al., 1993)". Moreover, as Solomon (2000) points out "quantifying the release rate and external input of ${ }^{4} \mathrm{He}$ are typically the largest challenges in the quantitative use of ${ }^{4} \mathrm{He}$ as a groundwater dating tool". Therefore, there is a need for additional data on helium input from the crust and mantle at different parts of the world.

Konya Closed Basin (KCB) in central Turkey, hosting a large confined/unconfined aquifer system with groundwater travel times up to 40,000 years (e.g., Bayari et al., 2009a), was selected as a field site to observe the variation of dissolved noble gas concentrations along regional groundwater flow path (Ozyurt \& Bayari, 2015). Results indicate a strong contribution from crust and mantle while atmospheric input is of secondary importance. Strong mantle and crustal gas input also explains the source of extensive carbon-dioxide dissolved in groundwater that leads to gigantic collapse doline formations developed in carbonate rocks (Bayari et al., 2009b) and shed light on the importance of emerging hypogene karst development theory in the KCB (Ozyurt \& Bayari, 2014, 2015).

\section{THE STUDY AREA}

Konya Closed Basin (KCB), located in central Anatolia (Turkey), is one of the major $\left(>50,000 \mathrm{~km}^{2}\right)$ endorheic basins in the world (Fig. 1). The mean elevation increases from about $900 \mathrm{~m}$ at the Salt Lake up to 2,000 $\mathrm{m}$ at the water divide along Taurus Mountains at the south. Currently, a semi-arid climate, with estimated mean annual precipitation of $450 \mathrm{~mm}$ and potential evapotranspiration of $1,400 \mathrm{~mm}$ dominates the KCB.

A simplified tectono-stratigraphic sequence in the KCB includes, from bottom to top a) PaleozoicMesozoic aged basement rocks, b) Paleogene, c) Neogene and d) Plio-Quaternary rocks (Fig. 2). The Paleozoic-Mesozoic is represented mainly by carbonate rocks exposed in the Taurus Mountains at the south and in the Obruk Plateau which separates the KCB into northern and southern sub-basins. The Paleogene rocks comprise detrital, carbonate, evaporitic and volcanic rocks while the Neogene sequence involves mainly lacustrine-detrital and lacustrine-carbonate sediments. Terrestrial PlioQuaternary sediments are made up mainly of alluvial fans and detrital lake deposits. The Plio-Quaternary lake deposits are practically impermeable and cover the surface of northern and southern sub-basins. The underlying Neogene lacustrine carbonate-rich rocks with well-developed karst constitute the main freshwater aquifer in the basin.
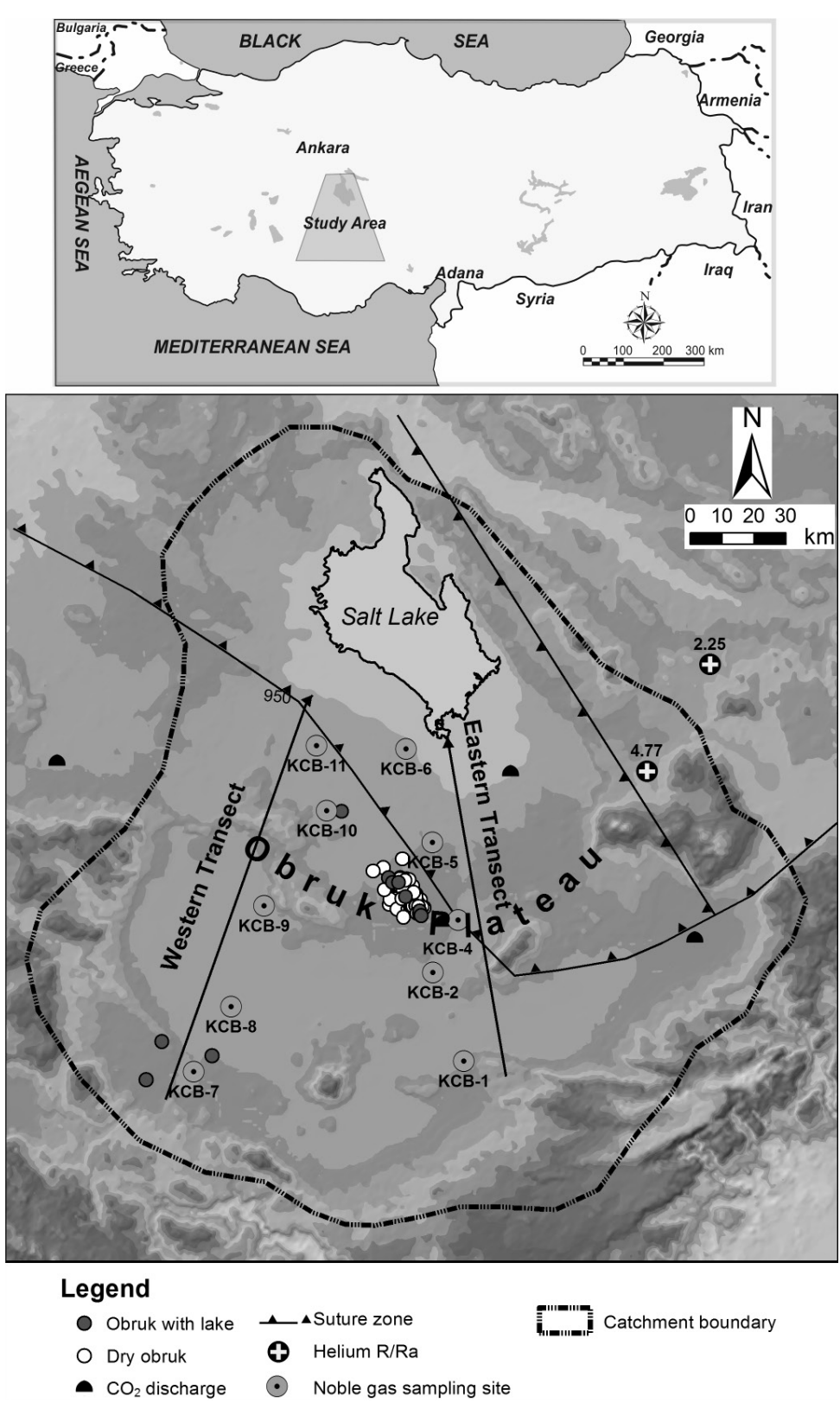

Fig. 1. Characteristics and noble gas sampling points of the study area (modified after Bayari et al., 2009b).

Groundwater in Neogene aquifer is the primary water resource in $\mathrm{KCB}$. The aquifer is fed mainly from the Taurus Mountains located at south from where the groundwater flows towards to the terminal Salt Lake at north (Bayari et al., 2009a, b). The regional groundwater flow system in between the main recharge and discharge areas is confined from top by fine-grained Plio-Quaternary lake bottom sediments except in the halfway where it becomes temporarily unconfined along the E-W trending Obruk Plateau of carbonate rocks. Time of travel of groundwater from the foot of Taurus Mountains towards the Salt Lake takes more than 40,000 years, based on radiocarbon dating, which is also supported by hydraulic ages (Bayari et al., 2009a).

The Obruk Plateau is abound with obruks. Obruk (e.g., UNESCO, 1972) is the Turkish name of gigantic karst collapse dolines with depths and diameters reaching $>200 \mathrm{~m}$ and $>300 \mathrm{~m}$ which have been formed by the dissolution of carbonate rocks by the extensive hypogenic carbon-dioxide input. According to Bayari et al. (2009b), "presence of volcanogenic 


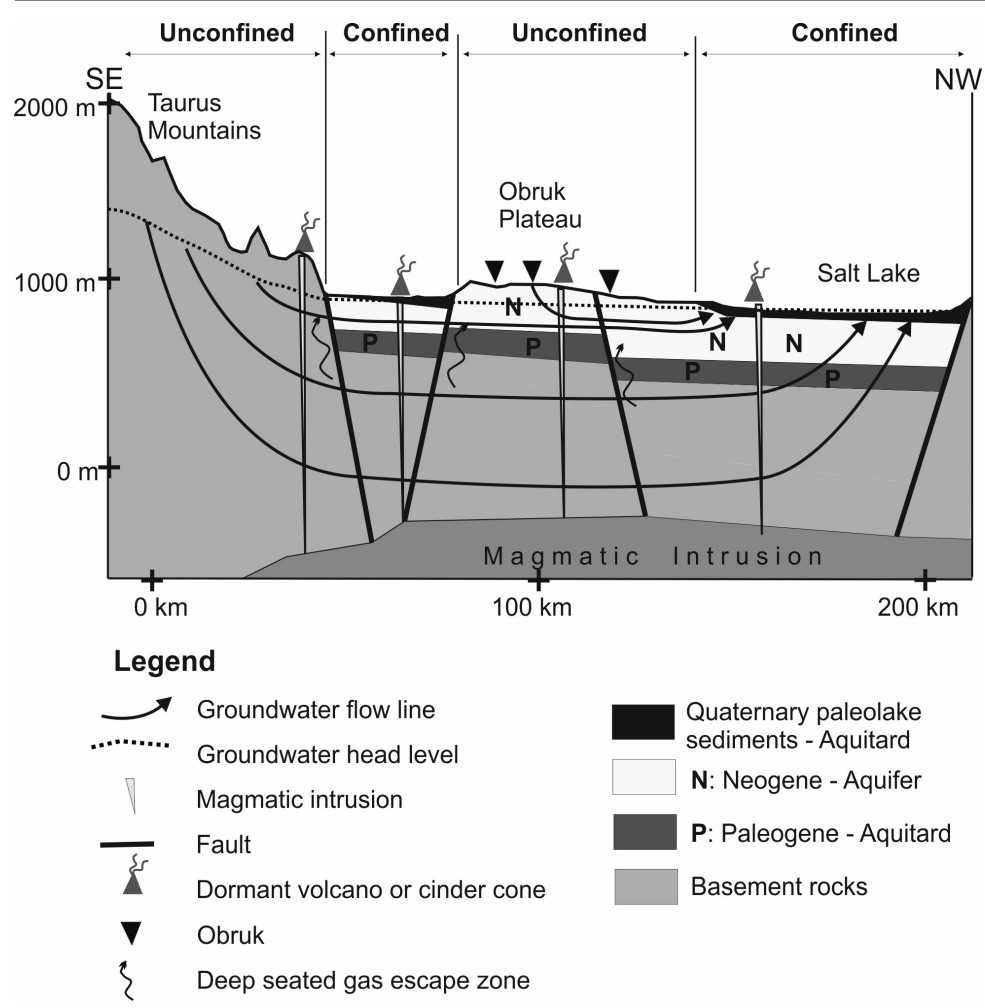

Fig. 2. Generalized geological cross-section of the study area (modified after Bayari et al., 2009b).

elements (i.e., Li and F) and remarkably high dissolved carbon dioxide $\left(\log \mathrm{PCO}_{2}=10^{-1}\right.$ atm) in groundwater, hydrothermal springs with elevated He contents (e.g., $\mathrm{R} / \mathrm{Ra}=4.77)$ and with highly enriched $-{ }^{13} \mathrm{C}$ of total dissolved inorganic carbon $\left(\delta^{13} \mathrm{C}\right.$ TDIC $=-1.12 \%$ o $\mathrm{V}-\mathrm{PDB}$ ) in the regional groundwater and presence of widespread carbon dioxide discharges constitute apparent evidence for the hypogenic fluid migration into the Neogene aquifer where enhanced dissolution due to mixing between the shallow-fresh and deepsaline groundwater gives rise to obruk formation" (Figs 1 and 2). Recent data on noble gases dissolved in regional groundwater supports the "geogenic" (i.e., both crust and mantle) origin for the dissolved carbondioxide input that leads to formation of these megascale karst depressions (Bayari et al., 2009b). Many of the obruks are located along a $70 \mathrm{~km}$-long NWSE trending belt which appears to mark the suture zone between Tauride-Anatolian and Sakarya Zone tectonic blocks of the Anatolian Plate. Thus, formation of obruks seems to be associated with the pointwise upwelling of carbon dioxide along this belt from the deep-rooted magmatism, which fed the Quaternary volcanic activity.

\section{DATA AND METHODS}

Samples from 10 deep wells along the eastern (samples 1 through 6 except, 3) and western transects (samples 7 through 11) that extend from the flank of Taurus Mountains (i.e., the main recharge area) to Salt Lake (i.e., the main discharge area) were collected on August 2011 to determine the noble gas, stable water isotopes (oxygen-18, deuterium) and tritium content of the groundwater along the regional flow path (see Fig. 1). All sampling wells were drilled previously by farmers extend at least up to $100 \mathrm{~m}$ depth from surface.
Therefore, considering the horizontal flow distance of about $120 \mathrm{~km}$ between the main recharge and discharge areas, the sampling depths in all wells do likely represent the same or close flow lines in the aquifer. Samples were collected from the wellhead by using the submerged pumps. Pumps were operated for a period to ensure that "fresh" groundwater is pumped out from the aquifer. Stability of temperature and specific conductance of groundwater are used to determine the arrival of fresh groundwater at the wellhead.

Duplicate samples for noble gas analyses were collected in copper tubes clamped at both ends. Samples for major ions, stable water isotopes and tritium analyses were taken into double-capped polyethylene bottles filled under water in a bucket to prevent air entrapment. Noble gas and tritium samples were analyzed at Noble Gas Laboratory of IAEA at Vienna. Measurement errors of the noble gas analyses range from $0.8 \%(\mathrm{Ne})$ to $4.5 \%$ $(\mathrm{Xn})$. Stable water isotopes of oxygen $\left(\delta^{18} \mathrm{O}\right)$ and hydrogen $\left(\delta^{2} \mathrm{H}\right)$ were analyzed at Sirfer Laboratory of University of Utah with an overall analytical uncertainty of $1 \%$ o $\mathrm{V}$-SMOW for $\delta^{2} \mathrm{H}$ and $0.1 \%$ o $\mathrm{V}$-SMOW for $\delta^{18} \mathrm{O}$. Major ion concentrations were analyzed by high performance ion chromatography at the Water Chemistry Laboratory of Hacettepe University. Detection limits of major ions are better than $0.001 \mathrm{mg} / 1$ and the electro-neutrality of the analyses are better than 5\%. While all major ions have been analyzed only Li and F concentrations are presented in this paper. In-situ hydrochemistry measurements and results of laboratory analyses are given in Table 1.

\section{RESULTS AND DISCUSSION}

\section{Characterization of groundwater along transects}

Specific conductance (i.e., electrical conductivity at $25^{\circ} \mathrm{C}, \mathrm{SEC}$ ) and temperature of groundwater gradually increase along the groundwater flow path up to intermediate recharge zone in the Obruk Plateau (marked by KCB 5 and KCB 9 in Table 1) where many of the obruks are located. Then, SEC and temperature slightly decrease after the Obruk Plateau because of intermediate recharge that occurs in this area.

All groundwater samples are located along the global meteoric water line in the $\delta^{18} \mathrm{O}$ versus $\delta^{2} \mathrm{H}$ graph (Fig. 3). $\mathrm{KCB} 7$ is the most isotopically enriched groundwater among all samples. This water represents partly evaporated shallow groundwater which is recharged mostly by a stream draining the shallow lakes located at the lower altitudes of the northern flank of Taurus Mountains. The isotopic composition of this sample is closer to the long-term mean composition of GNIP (Global Network of Isotopes in Precipitation) Ankara station (GNIP ID: 1713000) which is located at $900 \mathrm{~m}$ a.s.1. at about $150 \mathrm{~km}$ northeast of the Salt Lake (Fig. 3). Stable isotope composition recorded at this station represents the precipitation falling on lower elevations of KCB. Samples of two transects form two separate groups on the $\delta^{18} \mathrm{O}$ versus $\delta^{2} \mathrm{H}$ plot. Isotopic compositions of the eastern transect samples 


\begin{tabular}{|c|c|c|c|c|c|c|c|c|c|c|c|c|c|c|}
\hline 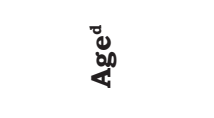 & 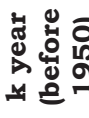 & & o & 6 & $\stackrel{\infty}{-}$ & $\stackrel{2}{2}$ & S & & $\infty$ & $\stackrel{-}{\sim}$ & $\stackrel{-}{2}$ & $\stackrel{\infty}{N}$ & ले & \\
\hline „əourłs! & : & & - & i & 농 & ๙ & $?$ & o & 우 & ০ & \& & ㄴ. & $\stackrel{\circ}{\circ}$ & \\
\hline 晃 & ஃ & & $\widehat{N}$ & $\frac{1}{5}$ & $\underset{\infty}{\infty}$ & $\stackrel{n}{7}$ & 17 & Ri & 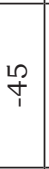 & $\hat{i}$ & ọ & $\stackrel{0}{1}$ & $\vec{i}$ & 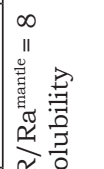 \\
\hline$\underset{\substack{0 \\
\infty \\
\infty}}{0}$ & ஃ。 & & $\begin{array}{l}9 \\
0 \\
7\end{array}$ & $\begin{array}{l}\text { ?े } \\
\text { ? } \\
1\end{array}$ & $\vec{F}$ & $\begin{array}{l}0 \\
0 \\
1\end{array}$ & $\begin{array}{l}9 \\
\vdots \\
\end{array}$ & ?o & $\begin{array}{l}\dot{\nabla} \\
\dot{\varphi}\end{array}$ & $\hat{i}$ & $\begin{array}{l}\stackrel{\circ}{\circ} \\
\stackrel{+}{1}\end{array}$ & $\begin{array}{ll}10 \\
9 \\
i\end{array}$ & $\begin{array}{l}0 \\
\stackrel{i}{i}\end{array}$ & 量玄 \\
\hline 田葋 & $\begin{array}{c}0 \\
+1 \\
+1\end{array}$ & & $\approx$ & $\begin{array}{l}\circ \\
\stackrel{0}{0}\end{array}$ & : & $\bar{c}$ & 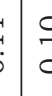 & 0 & $\begin{array}{c}\hat{N} \\
\text { ஸे }\end{array}$ & $\begin{array}{l}\circ \\
\stackrel{-}{\circ}\end{array}$ & $\begin{array}{l}0 \\
0 \\
0\end{array}$ & $\overrightarrow{7}$ & $\vec{z}$ & a \\
\hline 㸵 & P & & ले & O & $\stackrel{\mathrm{T}}{\mathrm{0}}$ & $\begin{array}{l}0 \\
0\end{array}$ & & \begin{tabular}{l}
$\infty$ \\
\hdashline \\
0 \\
\end{tabular} & $\begin{array}{l}\stackrel{p}{+} \\
\text { in }\end{array}$ & 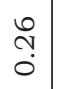 & $\stackrel{0}{\circ}$ & $\begin{array}{ll}n \\
0 \\
-i\end{array}$ & $\begin{array}{l}\hat{N} \\
\stackrel{-}{-}\end{array}$ & \\
\hline$\ddot{x}$ & \multirow{6}{*}{ 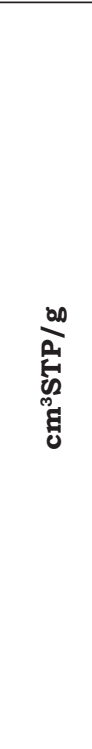 } & $\stackrel{\sim}{\sim}$ & $\underset{\approx}{\tilde{z}}$ & $\begin{array}{l}\stackrel{\infty}{ } \\
\stackrel{1}{0} \\
\stackrel{0}{0} \\
\stackrel{\sim}{\sim}\end{array}$ & $\stackrel{+}{\infty}$ & $\begin{array}{l}\infty \\
0 \\
0 \\
0 \\
1 \\
7\end{array}$ & 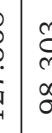 & $\begin{array}{l}m \\
m \\
0 \\
\infty \\
0 \\
0\end{array}$ & 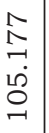 & 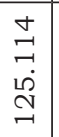 & $\begin{array}{l}\text { ồ } \\
\text { o. } \\
\dot{\vec{T}}\end{array}$ & 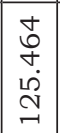 & 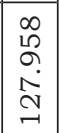 & $\frac{9}{2}$ \\
\hline 苞 & & ন্ं & $\begin{array}{l}\infty \\
\infty \\
\infty \\
\infty \\
\infty\end{array}$ & $\begin{array}{l}N \\
w \\
\infty \\
\infty \\
\infty\end{array}$ & $\begin{array}{l}\hat{N} \\
\text { ò } \\
\text { م }\end{array}$ & $\mid \begin{array}{l}\infty \\
\infty \\
\alpha \\
\alpha \\
1\end{array}$ & î & $\begin{array}{l}0 \\
\vec{y} \\
i \\
i\end{array}$ & $\begin{array}{l}0 \\
\text { L } \\
0 \\
\dot{0} \\
\infty\end{array}$ & $\begin{array}{l}0 \\
\infty \\
0 \\
\dot{+} \\
\infty\end{array}$ & $\begin{array}{l}\infty \\
\infty \\
\infty \\
\dot{\infty}\end{array}$ & 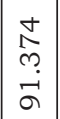 & 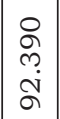 & \\
\hline 进㕵 & & $\begin{array}{l}\sigma \\
\sigma \\
\infty \\
\infty\end{array}$ & $\mid \begin{array}{l}\infty \\
\infty \\
\sim \\
0 \\
e\end{array}$ & $\begin{array}{l}= \\
\sigma \\
\infty \\
\infty \\
0\end{array}$ & $\begin{array}{l}\infty \\
0 \\
\infty \\
0 \\
0\end{array}$ & $\begin{array}{l}n \\
\stackrel{n}{n} \\
2 \\
\end{array}$ & 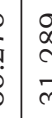 & 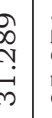 & 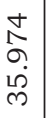 & $\begin{array}{l}\hat{\sigma} \\
\infty \\
\dot{0} \\
0\end{array}$ & $\begin{array}{l}\stackrel{+}{+} \\
\stackrel{+}{+}\end{array}$ & $\mid \begin{array}{l}\infty \\
o \\
\hat{\alpha} \\
\dot{\gamma} \\
q\end{array}$ & 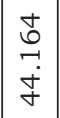 & \\
\hline 芒 & & $\begin{array}{l}\qquad \\
0 \\
0 \\
i\end{array}$ & $\frac{9}{9}$ & $\begin{array}{l}\overrightarrow{\text { in }} \\
\text { c } \\
\text { i }\end{array}$ & $\stackrel{-}{N}$ & สิ & ?ִ & $\begin{array}{ll}c \\
c \\
c \\
-i\end{array}$ & $\begin{array}{l}\infty \\
\stackrel{\infty}{*} \\
\vec{i}\end{array}$ & 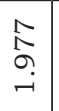 & 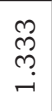 & 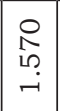 & \begin{tabular}{|c|}
$\infty$ \\
$N$ \\
\\
$-i$ \\
\end{tabular} & \\
\hline 承 : & & & $\mid \begin{array}{l}\infty \\
m \\
0\end{array}$ & 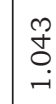 & 只 & $\begin{array}{l}0 \\
7 \\
0\end{array}$ & & $\begin{array}{c}\stackrel{+}{0} \\
\stackrel{n}{0} \\
0\end{array}$ & $\begin{array}{l}0 \\
0 \\
0 \\
0\end{array}$ & 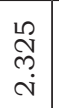 & 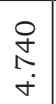 & \begin{tabular}{|l|} 
\\
0 \\
0 \\
$\infty$ \\
$\infty$
\end{tabular} & 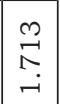 & \\
\hline 舫 & & \begin{tabular}{l}
$\frac{1}{2}$ \\
\multirow{2}{*}{} \\
0
\end{tabular} & mू & 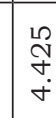 & $\begin{array}{l}2 \\
8 \\
0 \\
0 \\
0\end{array}$ & 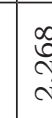 & & $\begin{array}{l} \\
\text { L } \\
\text { L } \\
\text { in }\end{array}$ & \begin{tabular}{c}
\multirow{2}{*}{} \\
$\hat{L}$ \\
0 \\
0
\end{tabular} & 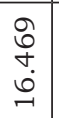 & 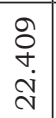 & \begin{tabular}{|l|}
$\infty$ \\
$\infty$ \\
$\infty$ \\
$\infty$ \\
$\sim$ \\
$\sim$
\end{tabular} & \begin{tabular}{|l|}
$\vec{N}$ \\
$\infty$ \\
$\dot{1}$ \\
$\dot{\omega}$
\end{tabular} & \\
\hline 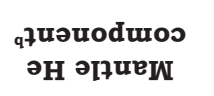 & $\delta^{\circ}$ & & $\begin{array}{l}\infty \\
+ \\
+\end{array}$ & $\hat{\sigma}$ & $\overrightarrow{0}$ & I & & $\overrightarrow{0}$ & $\stackrel{\sim}{i}$ & $\overrightarrow{\vec{i}}$ & $\begin{array}{l}\infty \\
\infty \\
\infty \\
\sim\end{array}$ & \begin{tabular}{l|} 
\\
$\stackrel{N}{j}$ \\
v
\end{tabular} & 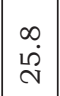 & \\
\hline $\begin{array}{l}\text { qquouoduros } \\
\text { әH [e7snxכ }\end{array}$ & $\therefore$ & & & $\begin{array}{l}0 \\
\infty \\
\infty\end{array}$ & $\overrightarrow{0}$ & $\begin{array}{l}m \\
0 \\
0 \\
0\end{array}$ & $\begin{array}{l}\dot{b} \\
\dot{b} \\
b \\
a \\
a\end{array}$ & $\begin{array}{l}\infty \\
\infty \\
0 \\
0\end{array}$ & 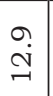 & $\begin{array}{l}\overrightarrow{1} \\
\dot{\infty} \\
\infty\end{array}$ & $\begin{array}{l}\text { aे } \\
\text { ò }\end{array}$ & $\begin{array}{l}\stackrel{1}{L} \\
\dot{L}\end{array}$ & $\begin{array}{ll} \\
\infty \\
0 \\
0\end{array}$ & \\
\hline 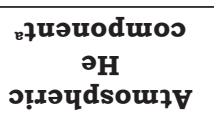 & $\circ^{\circ}$ & & 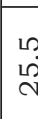 & ^ & $\stackrel{\infty}{\infty}$ & $\begin{array}{l}n \\
0 \\
0\end{array}$ & & $\begin{array}{l}\stackrel{0}{\rho} \\
\stackrel{\rho}{\rho}\end{array}$ & $\begin{array}{l}\sigma \\
\dot{+} \\
\infty\end{array}$ & $\begin{array}{l}\infty \\
i\end{array}$ & $\stackrel{+}{\sim}$ & $\hat{i}$ & $\begin{array}{l}0 \\
0\end{array}$ & \\
\hline$\frac{\mathscr{\alpha}}{\text { ณ }}$ & & & In & 동 & $\mid \begin{array}{l}\infty \\
\infty \\
0 \\
0\end{array}$ & m & & $\begin{array}{l}0 \\
+ \\
- \\
-\end{array}$ & $\stackrel{\vec{H}}{-}$ & 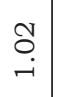 & 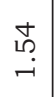 & $\begin{array}{c}+ \\
\infty \\
-i \\
-i\end{array}$ & $\begin{array}{c}m \\
\vec{i} \\
i\end{array}$ & \\
\hline E & $\overrightarrow{\text { bo }}$ & & $\frac{0}{2}$ & $\begin{array}{l}8 \\
\text { ले } \\
0\end{array}$ & $\begin{array}{l}0 \\
\infty \\
0 \\
0\end{array}$ & $\begin{array}{l}\infty \\
\infty \\
0 \\
0\end{array}$ & $\begin{array}{ll}0 \\
b \\
b \\
b \\
\vdots\end{array}$ & 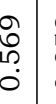 & $\begin{array}{c}0 \\
\stackrel{0}{0} \\
m \\
0\end{array}$ & $\begin{array}{l}\overrightarrow{3} \\
0 \\
0\end{array}$ & $\begin{array}{l}8 \\
2 \\
0\end{array}$ & 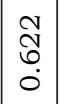 & $\begin{array}{l}0 \\
\text { Dे } \\
\text { i? } \\
0\end{array}$ & \\
\hline$ت$ & $\underset{\mathrm{ab}}{\overrightarrow{\mathrm{b}}}$ & & 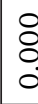 & oे & 㝵 & $\mid \begin{array}{l}\infty \\
n \\
n \\
0 \\
0\end{array}$ & & \begin{tabular}{l|l}
0 \\
$\overrightarrow{7}$ \\
0 \\
0
\end{tabular} & $\begin{array}{l}\dot{1} \\
\dot{0} \\
0 \\
0\end{array}$ & $\begin{array}{l}\stackrel{1}{0} \\
9 \\
\stackrel{0}{0}\end{array}$ & $\begin{array}{l}0 \\
\stackrel{0}{0} \\
\stackrel{1}{0} \\
0\end{array}$ & $\begin{array}{l}1 \\
\overrightarrow{0} \\
0\end{array}$ & $\begin{array}{l}1 \\
0 \\
0 \\
0 \\
0\end{array}$ & \\
\hline $\begin{array}{l}0 \\
\text { 界 }\end{array}$ & $\frac{a}{20}$ & & $\frac{A}{2}$ & 占 & $\stackrel{๑}{\beth}$ & $\stackrel{0}{1}$ & & હે & 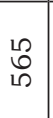 & $\begin{array}{l}0 \\
\infty \\
\stackrel{ }{-1}\end{array}$ & $\begin{array}{l}8 \\
0 \\
-1\end{array}$ & $\begin{array}{c}\infty \\
\infty \\
\infty \\
\sim\end{array}$ & î̀ & \\
\hline 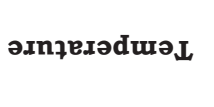 & 0 & $\stackrel{\circ}{\sim}$ & in & \pm & $\stackrel{\text { L }}{ \pm}$ & वे & לִ & 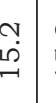 & $\begin{array}{l}\stackrel{\sigma}{\circ} \\
\stackrel{n}{न}\end{array}$ & 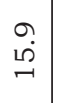 & $\begin{array}{l}0 \\
\dot{\mathrm{d}}\end{array}$ & $\begin{array}{l}\circ \\
\dot{9}\end{array}$ & $\stackrel{m}{\stackrel{m}{\sim}}$ & \\
\hline 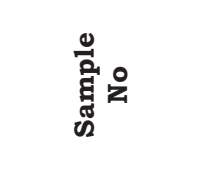 & & 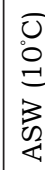 & $\vec{m}$ & $m$ & $\stackrel{+}{\stackrel{m}{0}}$ & 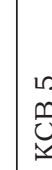 & 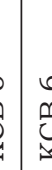 & $\begin{array}{l}0 \\
m \\
0 \\
\end{array}$ & 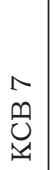 & \begin{tabular}{l}
$\infty$ \\
$m$ \\
0 \\
\multirow{y}{*}{}
\end{tabular} & \begin{tabular}{l}
$a$ \\
$m$ \\
0 \\
\multirow{y}{*}{}
\end{tabular} & \begin{tabular}{c}
0 \\
$\vec{m}$ \\
0 \\
\hdashline \\
\hdashline 1
\end{tabular} & $\begin{array}{l}\overrightarrow{\vec{p}} \\
\overrightarrow{0} \\
\dot{y}\end{array}$ & \\
\hline
\end{tabular}




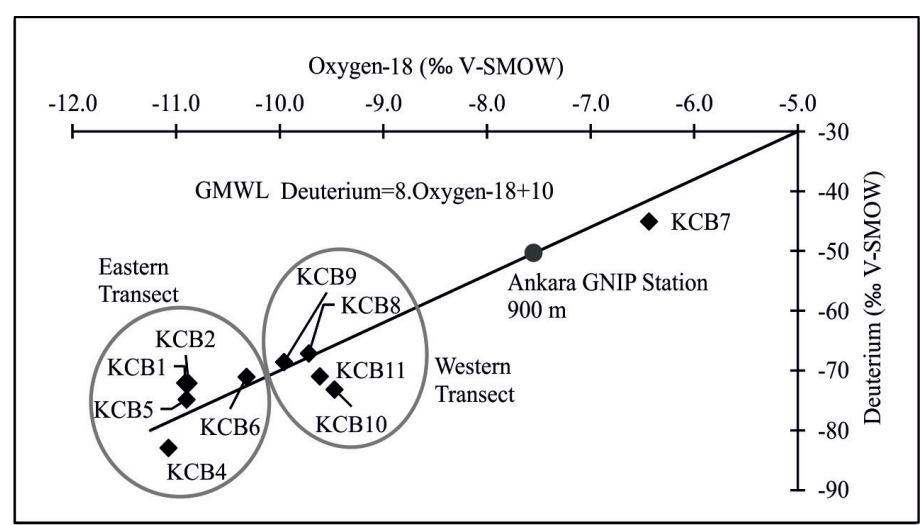

Fig. 3. Stable isotope scatter plot of groundwater samples.

are more depleted than the samples of western transect probably because of the lower mean recharge temperature at higher elevations. As seen from the relief on Figure 1, samples of eastern transect are recharged from higher elevations compared to the samples of western transect. Bayari et al (2009a) found that the groundwater's $\delta^{18} \mathrm{O}$ value at the flank of Taurus Mountains (main recharge area) is lower to more than $2 \%$ near the Salt Lake (main discharge area). The radiocarbon ages of groundwater ranges from recent to about 40,000 years before present (i.e., 1950) at the mountain flank and near the Salt Lake, respectively. Therefore, such a drop in $\delta^{18} \mathrm{O}$ content is in agreement with an expected mean annual groundwater recharge temperature drop of $6-8^{\circ} \mathrm{C}$ between Holocene inter-glacial and Pleistocene glacial climates.

However, a similar trend is not observed along transects of this study. The mismatch between the $\delta^{18} \mathrm{O}$ and $\delta^{2} \mathrm{H}$ data sets of Bayari et al (2009a) and this study is probably associated with the different well/filter depths sampled particularly at the end of transects. In other words, the shallower the filter depth, the younger the groundwater which has more enriched $\mathrm{\delta}^{18} \mathrm{O}$ signal.

Tritium values along both transect

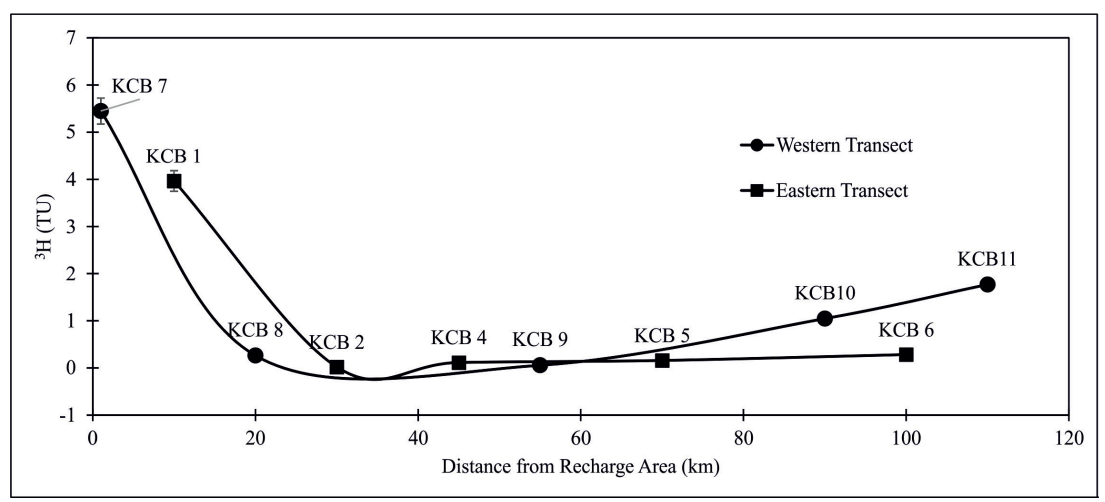

Fig. 4. Tritium content of groundwater along the flow transects.

geochemical model that is based on a "geogenic" $\mathrm{CO}_{2}$ component with $\delta^{13} \mathrm{C}=0 \%$ V-PDB and ${ }^{14} \mathrm{C}=0$ pmc. After testing all likely end-member sources, they come up with the conclusion that a "geogenic" (i.e., crustal and/or mantle) gas input must occur to explain the high dissolved carbon-dioxide concentrations and the associated ${ }^{13} \mathrm{C}$ signal in groundwater. The ${ }^{14} \mathrm{C}$ and ${ }^{3} \mathrm{H}$ signals agree along the regional flow path and the ${ }^{14} \mathrm{C}$ ages calculated for the downstream of Obruk Plateau account for the effect of atmospheric carbondioxide input supplied by the intermediate recharge. Groundwater's radiocarbon ages are correlated well also with the hydraulic ages which increase by the increasing distance from the main recharge area, Taurus Mountains (Bayari et al., 2009a).

\section{Discrimination of gas sources}

Measured ${ }^{4} \mathrm{He}, \mathrm{Ne}, \mathrm{Ar}, \mathrm{Kr}$, and Xe gas compositions and ${ }^{3} \mathrm{He} /{ }^{4} \mathrm{He}$ ratios of the samples collected along both transects are given in Table 1. Measured dissolved gas concentrations have not been corrected against the excess atmospheric component. This is because first, any correction scheme should be based on some assumptions which may not be validated and secondly, all samples along the both transects originate from the same recharge area where excess air inclusion should be the same for all samples if exists at all. vary similarly between $0.06 \mathrm{TU}$ and $5.45 \mathrm{TU}$ (see Table 1). In both transects, highest tritium contents are observed in samples, which are close to main recharge area. Then, tritium content rapidly decreases down to the detection limit of liquid scintillation counting, within $20-30 \mathrm{~km}$ from recharge area (Fig. 4). A slight increase in tritium content at western transects shows up beyond the Obruk Plateau as a result of young recharge that occurs in this area. The regional groundwater flow is practically tritium free except, samples $\mathrm{KCB} 1,7,10$, and 11 that possess young recharge. Such tritium isotope signal supports the model of a confined groundwater flow system with considerably long residence time in the $\mathrm{KCB}$.

Radiocarbon age-dating has been conducted by Bayari et al (2009a) on groundwater samples collected from the wells located nearby the sampling locations used in this study. Radiocarbon dates gradually increase along the regional groundwater flow path from recent at the mountain flank to about 40,000 years near the Salt Lake. Bayari et al (2009a) used a
Besides excess air involvement, degassing to atmosphere may occur during groundwater circulation (e.g., Mazor \& Bosch, 1992) or sampling. To investigate the presence of degassing, the ASW (Air Saturated Water) values are compared to measured gas concentrations (see Table 1). The ASW values are calculated for $10^{\circ} \mathrm{C}$ and $1,000 \mathrm{~m}$, which are the recent recharge temperature and mean recharge elevation of the Neogene aquifer.

A graph of ASW normalized noble gas contents except ${ }^{4} \mathrm{He}$ is given in Fig. 5 . A copy of graph with ${ }^{4} \mathrm{He}$ is given as inset in the same figure. As seen from the graph, in some of the samples all noble gasses are plotted below the ASW line whereas in some samples only Ne or Ar is plotted above the ASW. Gasses plotted below the ASW clearly indicate that outgassing during the sampling or in the aquifer has affected the original dissolved gas composition. Ne and Ar compositions above ASW may be associated with the errors that occurred at some stage of the overall analytical process. The ASW normalized ${ }^{4} \mathrm{He}$ plots on Fig. 5 


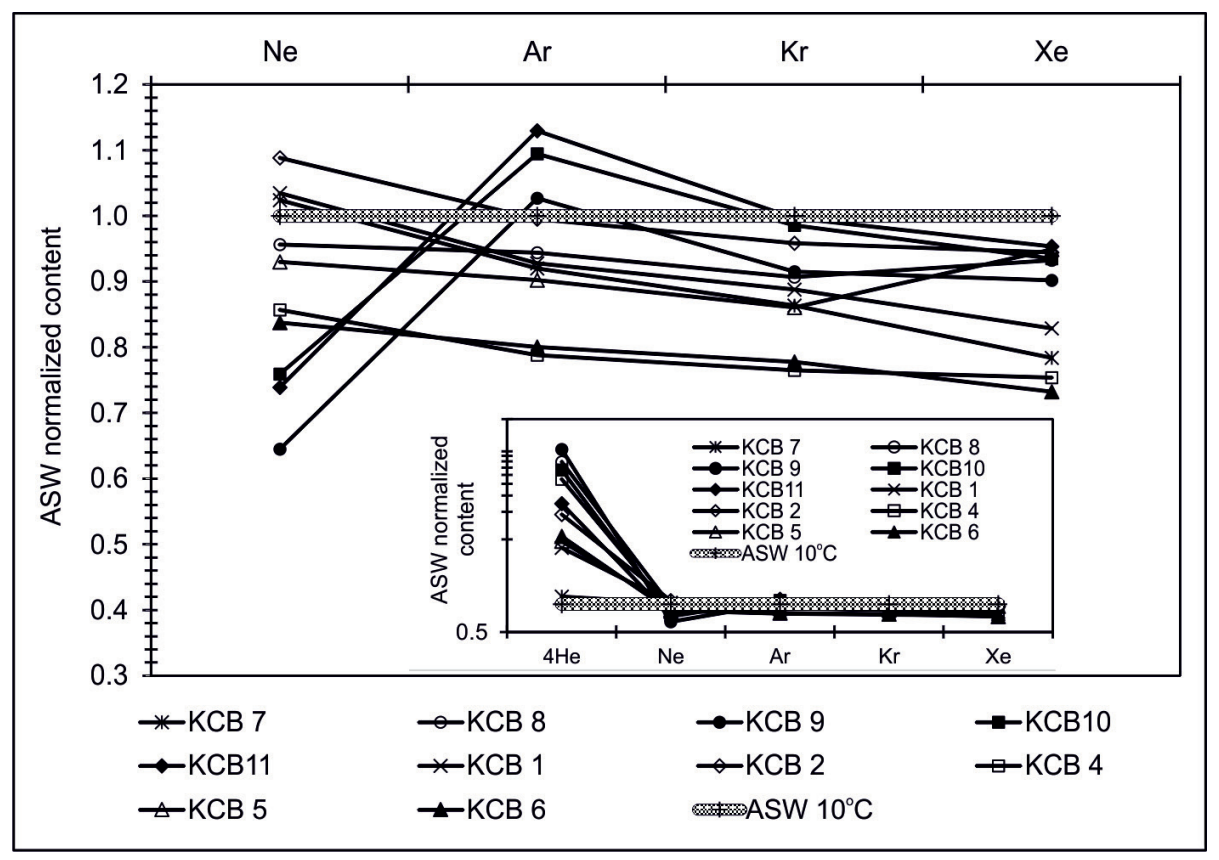

Fig. 5. ASW-normalized noble gas contents.

indicate that the samples have ${ }^{4} \mathrm{He}$ concentrations ranging between 5 to 50 times of ASW. In other words, even if some of the initial dissolved gas contents of the samples are lost, the very high ${ }^{4} \mathrm{He}$ content of the samples apparently imply presence of $\mathrm{He}$ sources other than the atmosphere.

A scatter plot of $\mathrm{Ne} / \mathrm{He}$ versus $\log { }^{3} \mathrm{He} /{ }^{4} \mathrm{He}$ is used to discriminate the gas sources in the samples (Fig. 6). For all samples, the likely sources of gasses (i.e., end-members) are atmosphere, crust and mantle. Atmosphere is the major source of dissolved $\mathrm{Ne}$ in groundwater (e.g., Solomon, 2000) so that increasing $\mathrm{Ne} / \mathrm{He}$ ratio implies increasing amount of atmospheric component in the sample. Samples with no or negligible atmospheric component has a $\mathrm{Ne} / \mathrm{He}$ ratio around zero. Unlike $\mathrm{Ne}$, dissolved $\mathrm{He}$ may originate from one or any combination of the atmospheric, crustal and mantle sources. However, ${ }^{3} \mathrm{He} /{ }^{4} \mathrm{He}$ ratio can be used to discriminate the source of dissolved He. ${ }^{4} \mathrm{He}$ produced mainly in crust through U-Th series decay and ${ }^{3} \mathrm{He}$ is released mainly from mantle and from radioactive decay of ${ }^{3} \mathrm{H}$. The typical ${ }^{3} \mathrm{He} /{ }^{4} \mathrm{He}$ ratios of atmosphere, crust and mantle are $1.38 \times 10^{-6}$ (Clarke et al., 1976), between $10^{-9}$ and $10^{-7}$ (typically $2.0 \times 10^{-8}$, Mamyrin \& Tolstikhin, 1984) and between $1.1 \times 10^{-5}$ and $1.4 \times 10^{-5}$ (Ozima \& Podosek, 1983), respectively. Available data do not allow to decide on whether the source of helium is upper mantle or lower mantle. On the other hand, the $\mathrm{Ne} / \mathrm{He}$ ratio of air saturated water at $10^{\circ} \mathrm{C}$ (ASW at $10^{\circ} \mathrm{C}$ ) is 4.34 . Figure 6 shows that the sample $\mathrm{KCB} 7$, located at the beginning of western transect has the highest atmospheric gas contribution as implied by its high $\mathrm{Ne} / \mathrm{He}$ ratio which is very close to atmospheric equilibrium ratio (i.e., ASW at $10^{\circ} \mathrm{C}$ ). Sample KCB-7 appears to be fed from a nearby stream originating from a dam located $14 \mathrm{~km}$ upstream and the mean annual recent recharge/air temperature of $10^{\circ} \mathrm{C}$ may be assumed. Therefore, a Ne/He value, which is identical to that of ASW, is expected for the sample KCB-7. Leftward deviation of the sample KCB7 from the ASW point may be due to outgassing during the sampling because relatively high degassing of $\mathrm{He}$ compared to $\mathrm{Ne}$ would reduce the $\mathrm{Ne} / \mathrm{He}$ ratio. The ASW normalized ${ }^{4} \mathrm{He}$ contents (see Table 1 and inset in Fig. 5) seem to have affected by the varying degree of outgassing because the horizontal scattering of the samples on Fig. 6 resembles the vertical scattering of ${ }^{4} \mathrm{He}$ on the inset of Figure 5 .

The samples other than KCB-7 show much limited atmospheric but remarkably high crustal and mantle components. Second highest $\mathrm{Ne} / \mathrm{He}$ ratio among all samples is observed in $\mathrm{KCB} 1$, which represents the groundwater in mountain flank at the beginning of eastern transect. It appears that, once

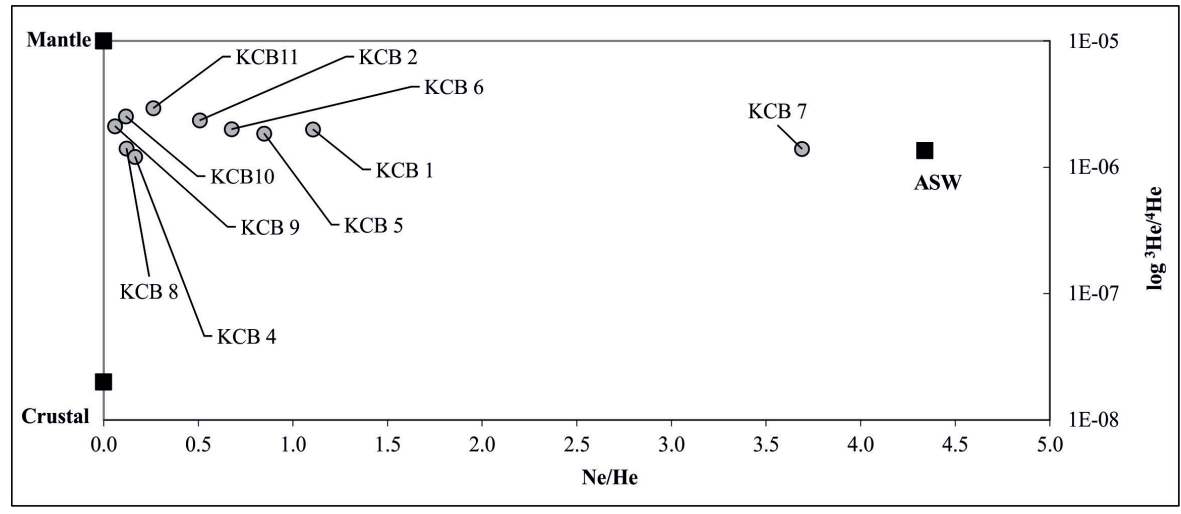

Fig. 6. Distribution of samples among likely end-members. 
the groundwater is isolated from atmosphere in the recharge area, increasing mantle and crustal gas input along the regional flow path reduces the $\mathrm{Ne} / \mathrm{He}$ ratio and the ${ }^{3} \mathrm{He} /{ }^{4} \mathrm{He}$ signal evolves depending on the helium isotope inputs. Fig. 5 indicates an input from crust, which is stronger than mantle input because the samples like $\mathrm{KCB} 2,4,8,9,10$, and 11 are plotted towards the crustal end-member. However, a mantle contribution cannot be neglected either.

$$
\left(\frac{N \mathrm{e}}{H e}\right)_{\text {Subsurface }} \cdot V_{\text {Subsurface }}+\left(\frac{N e}{H e}\right)_{\text {Atm }} \cdot V_{\text {Atm }}=\left(\frac{N e}{H e}\right)_{\text {Sample }} \cdot\left(V_{\text {Subsurface }}+V_{\text {Atm }}\right)
$$

where, the atmospheric $\mathrm{Ne} / \mathrm{He}$ ratio is 4.34 for ASW at $10^{\circ} \mathrm{C}$, subsurface $\mathrm{Ne} / \mathrm{He}$ ratio assumed equal to 0 because of negligible Ne production in the subsurface, $\mathrm{V}$ is the percent contribution to sample from subsurface $\left(\mathrm{V}_{\text {Subsurface }}\right)$ and atmospheric $\left(\mathrm{V}_{\mathrm{Atm}}\right)$ sources. Calculated atmospheric He contributions are presented in Table

$$
\left(\frac{R}{R_{a}}\right)_{\text {Mantle }} \cdot V_{\text {Mantle }}+\left(\frac{R}{R_{a}}\right)_{\text {Crust }} \cdot V_{\text {Crust }}=\left(\frac{R}{R_{a}}\right)_{\text {Sample }} \cdot\left(V_{\text {Mantle }}+V_{\text {Crust }}\right)
$$

where, $\mathrm{R}$ is the ${ }^{3} \mathrm{He} /{ }^{4} \mathrm{He}$ concentration ratio, $\mathrm{Ra}$ is the atmospheric ${ }^{3} \mathrm{He} /{ }^{4} \mathrm{He}$ ratio $\left(1.38 \times 10^{-6} \mathrm{~cm}^{3} \mathrm{STP} / \mathrm{g}\right), \mathrm{V}$ is the percent contribution to sample from mantle and crustal sources.

The ${ }^{3} \mathrm{He} /{ }^{4} \mathrm{He}$ ratios of the crust and mantle (i.e., MORB) are characterized by typical $\mathrm{R} / \mathrm{Ra}$ ratios of 0.05 and 8 , respectively. The $\mathrm{R} / \mathrm{Ra}$ ratios calculated for the KCB samples vary between 0.88 (KCB-4) and 2.13 (KCB-11). As seen from the values in Table 1 , both crustal and mantle sources contribute to the helium content of the groundwaters in KCB. The mantle gas contributions vary between 2 and 26\% among all samples of the transects studied. In the confined part between Taurus Mountains and the Obruk Plateau, the helium gas concentrations increase linearly. According to $\mathrm{R} / \mathrm{Ra}$ values, the mantle $\mathrm{He}$ input gradually increases from 2 to $19 \%$ along the western transect whereas, in the eastern transect, the mantle He concentration first increases from 18 to $21 \%$ the, then declines to $10 \%$ probably because of degassing to atmosphere.

Since the mantle and crustal helium isotopic compositions in the $\mathrm{KCB}$ are not known exactly general literature values of these end members are
1. Highest atmospheric gas component is calculated as $85 \%$ for $\mathrm{KCB}-7$ sample.

Rest of gas content other than atmosphere are split into mantle and crustal components on the basis of Equation 2.

\section{used to calculate the contributions. Hence, the results given above represent locally meaningful values. \\ Evolution of helium isotopes along the regional groundwater flow path}

Figure 7 shows the evolution of ${ }^{3} \mathrm{He}$ and ${ }^{4} \mathrm{He}$ concentrations along the western and eastern transects of regional groundwater flow path. Isotope concentrations increase steadily from the mountain flank to Obruk Plateau under the confined flow conditions along both transects. In the Obruk Plateau, between eastern transect samples KCB 4 and 5 and, western transect samples of KCB 9 and 10 the flow system becomes unconfined and is open to intermediate recharge from surface. At the end of the confined flow section between mountain flank and Obruk Plateau, both ${ }^{3} \mathrm{He}$ and ${ }^{4} \mathrm{He}$ reach their highest concentrations at sites KCB 9 (in western transect) and $\mathrm{KCB} 4$ (in eastern transect). Measured ${ }^{3} \mathrm{He}$ and ${ }^{4} \mathrm{He}$ concentrations along western transect are much greater than those observed along eastern transect (about 4.5 times greater for ${ }^{3} \mathrm{He}, 2$ times greater for $\left.{ }^{4} \mathrm{He}\right)$. This shows that there may be substantial differences between the ${ }^{3} \mathrm{He}$ and ${ }^{4} \mathrm{He}$ crustal/

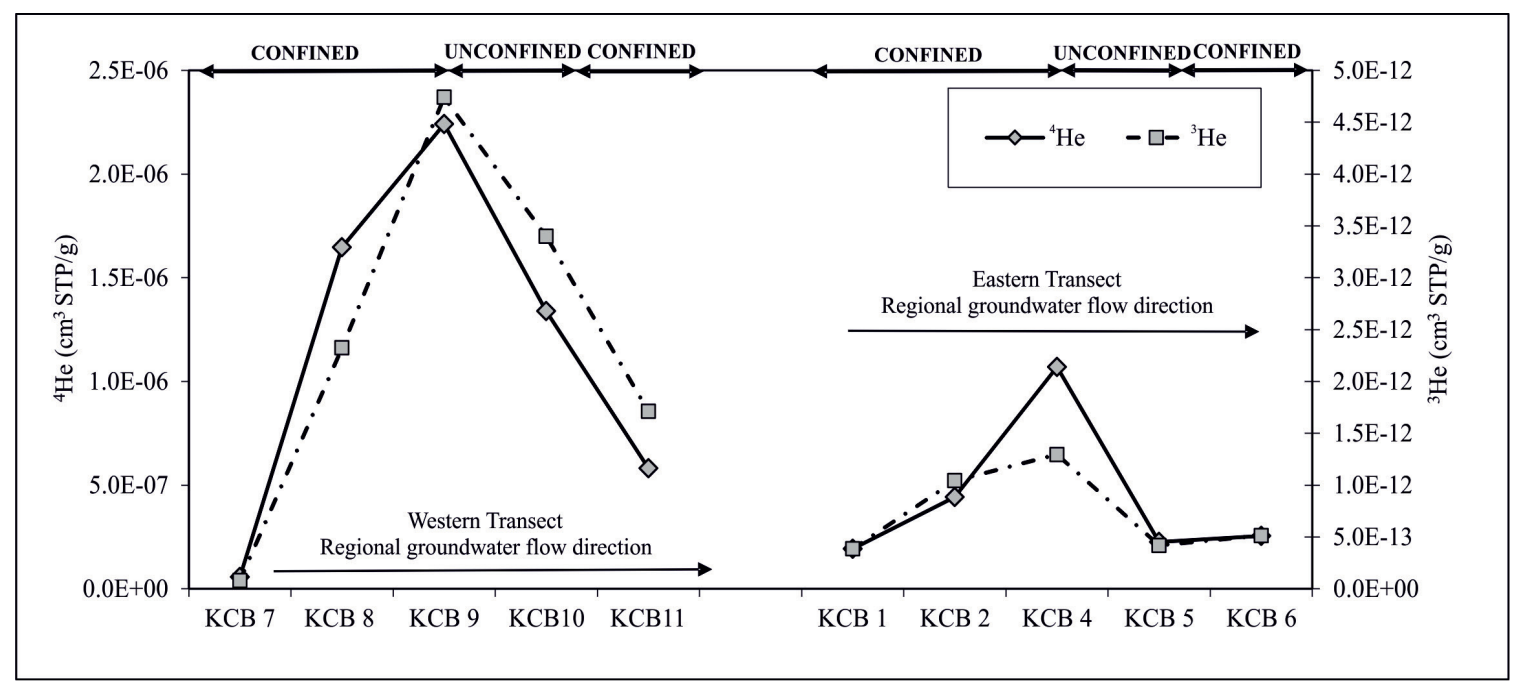

Fig. 7. Variation of dissolved $\mathrm{He}$ isotopes along the regional groundwater flow path. 
mantle inputs into the groundwater in different parts of a single aquifer system. Obviously, such spatial variations would complicate the use of ${ }^{4} \mathrm{He}$ as a groundwater dating tool (e.g., Solomon, 2000) because of the spatially varying He isotope input rates. For example, according to Bayari (2009a) the radiocarbon ages of groundwater around sites $\mathrm{KCB} 4$ $\left({ }^{4} \mathrm{He}=10.70 \times 10^{-7} \mathrm{~cm}^{3} \mathrm{STP} / \mathrm{g}\right)$ and $\mathrm{KCB} 9\left({ }^{4} \mathrm{He}=22.41\right.$ $\left.\times 10^{-7} \mathrm{~cm}^{3} \mathrm{STP} / \mathrm{g}\right)$ are about 18,000 and 14,000 years, respectively. At the beginning of these transects ${ }^{4} \mathrm{He}$ concentrations of recent groundwater are $1.93 \times 10^{-7}$ $\mathrm{cm}^{3} \mathrm{STP} / \mathrm{g}$ at KCB 1 and $0.57 \times 10^{-7} \mathrm{~cm}^{3} \mathrm{STP} / \mathrm{g}$ at KCB 7 . The corresponding radiocarbon ages at these sites are about 200 and 3,000 years, respectively. These numbers indicate ${ }^{4} \mathrm{He}$ accumulations of $8.77 \times 10^{-7}$ $\mathrm{cm}^{3} \mathrm{STP} / \mathrm{g}$ between sites $\mathrm{KCB} 1$ and 4 of eastern transect and $21.84 \times 10^{-7} \mathrm{~cm}^{3} \mathrm{STP} / \mathrm{g}$ between sites KCB 7 and 9 of western transect in a period of 17,800 and 11,000 years, respectively. Hence, the corresponding ${ }^{4} \mathrm{He}$ accumulation rates for eastern and western transect calculated as $4.93 \times 10^{-11} \mathrm{~cm}^{3} \mathrm{STP} / \mathrm{g} \bullet \mathrm{year}$ and $1.99 \times 10^{-10} \mathrm{~cm}^{3} \mathrm{STP} / \mathrm{g} \bullet$ year, respectively.

Figure 7 indicates that He isotope concentrations to the downstream of Obruk Plateau decline steadily along western transect but, first decrease and then slightly increase in the eastern transect. In general, this variation of He isotope concentrations is in agreement with the variation of dissolved volcanogenic elements like Li and F (Fig. 8). Like He isotopes, the $\mathrm{Li}$ and $\mathrm{F}$ ion concentrations gradually increase up to $\mathrm{KCB} 9$ and $\mathrm{KCB} 4$ sampling points.
Beyond these points, the Neogene aquifer in the Obruk Plateau becomes temporarily unconfined and the Li and F concentrations appear to have diluted by the intermediate recharge. The dilution of He isotope concentrations after the sites KCB 9 (on western transect) and KCB 4 (on eastern transect) should also be due to the intermediate recharge in the Obruk Plateau. The flow domain becomes confined again to the downstream of sites KCB 10 and 5 where no local recharge to groundwater occurs. Here, both ${ }^{3} \mathrm{He}$ and ${ }^{4} \mathrm{He}$ concentrations at site KCB 6 of eastern transect shows a slight increase. However, a similar trend is not observed at site $\mathrm{KCB} 11$ of western transect where both ${ }^{3} \mathrm{He}$ and ${ }^{4} \mathrm{He}$ concentrations continue to decline. Such a declining gas composition may be due to degassing to atmosphere through a thinning confining layer in this part of flow domain.

The SEC value of groundwater along eastern transect after the Obruk Plateau recharge zone (samples KCB 5 and 6) does not decrease as expected from a dilution by relatively "young" recharge and the ${ }^{3} \mathrm{H}$ data (e.g., KCB 6) does not indicate an apparent young groundwater contribution. Therefore, it is likely that the decreasing $\mathrm{He}$ concentrations at the end of eastern transect may be due mainly to degassing to atmosphere. On the other hand, decreasing SEC and increasing ${ }^{3} \mathrm{H}$ values (samples KCB-10 and 11 ) at the end of the western transect suggest that the dilution by relatively "young" recharge -along with the degassing to atmosphere- is more likely to be responsible for the decreasing He concentrations.

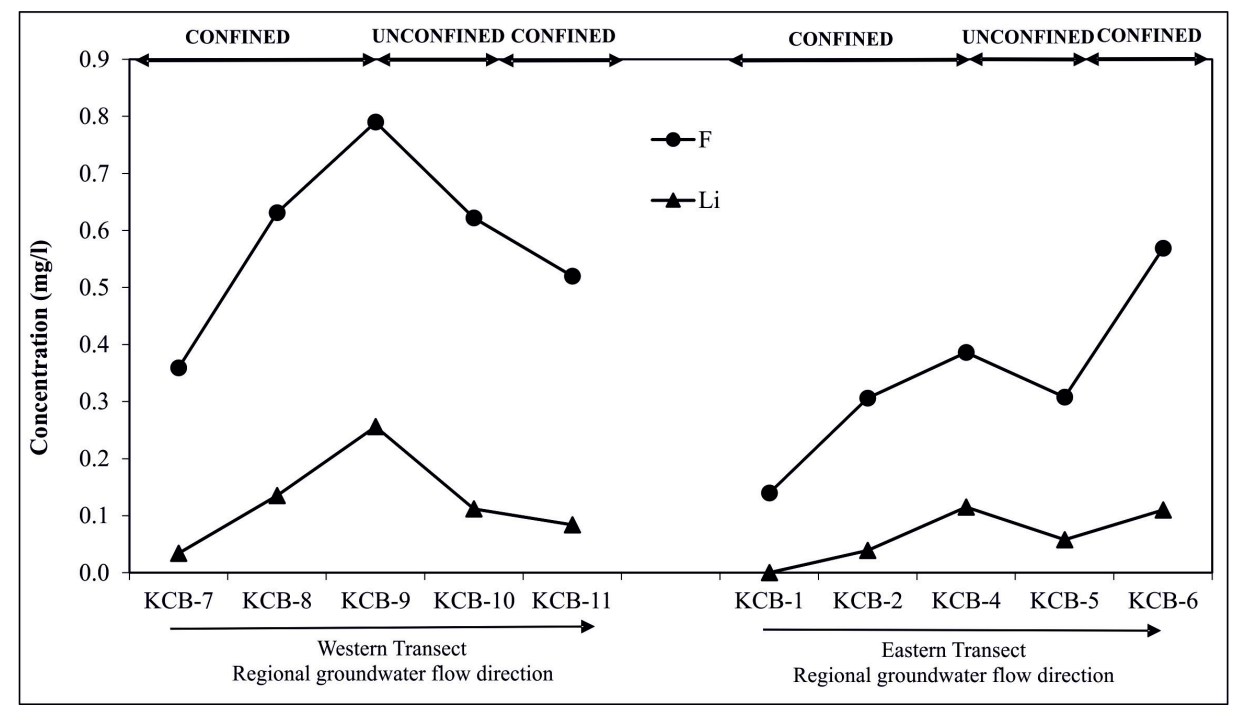

Fig. 8. Variation of $\mathrm{Li}$ and $\mathrm{F}$ concentrations along the regional groundwater flow path.

Eventually, the use of ${ }^{4} \mathrm{He}$ as a groundwater agedating tool seems to be practically limited as long as robust information regarding the local ${ }^{4} \mathrm{He}$ release rate exists. Data presented in this study shows that the ${ }^{4} \mathrm{He}$ accumulation rates may be at least four times variable among sites located 30 to $60 \mathrm{~km}$ apart. Temporal variations in the He accumulation in the Neogene aquifer may be due to i) some tectonic effects like buried faults zones with high permeability, ii) 3-dimensional variations in the permeability of aquifer/aquitard units, iii) presence of upwelling fluid spots at deeper parts, iv) spatially variable degassing to atmosphere due to varying thickness of confining layer, v) spatial variations in the concentrations of He-producing elements in the crust. Available information in the $\mathrm{KCB}$ is not sufficient to make a robust assessment on the factors affecting temporal dissolved helium variations in the groundwater.

\section{He isotopes as evidence of hypogenic karst development}

Karst systems are important water and oil/ gas reservoirs because of their extreme porosity and hydraulic conductivity development. Until recently, development of karst has been attributed primarily to epigenic dissolution (i.e., dissolution by 
acidic waters percolating from surface downwards). Biogenic carbon-dioxide dissolved in root-zone water is regarded as the main source of acidity required for epigenic dissolution. However, a recent paradigm shift in theory of karst development (e.g., Klimchouk, 2015) suggests that hypogenic karst development may be as common as epigenic karstification. Therefore, there is considerable global effort to demonstrate to what extend hypogenic processes have been effective in development of karst in any particular area in the world. In this context, the obruks in $\mathrm{KCB}$ appear as potential candidates of a unique hypogenic karst development (e.g., Waltham, 2015). Bayari et al. (2009a) used geologic, tectonic, geophysical, hydrochemical and carbon isotope data to propose a hypogenic karst development model for the formation of large scale collapse dolines in the KCB. However, none of the evidences -including ${ }^{13} \mathrm{C}$ TDIC signal around $0 \% \mathrm{~V}-\mathrm{PDB}$ are entirely convincing for the source of upwelling deep-seated carbon-dioxide model as the main driver of hypogenic karstification in the KCB. Instead, $\mathrm{He}$ isotope signal obtained from regional groundwater samples unequivocally indicates a combined crustal and mantle source for the deep-seated gas input into the aquifer. Therefore, a hypogenic karst development model driven mainly by crustal and partly by mantle carbon-dioxide seems very convincing and complements the likely hypothesis that the development of obruks is the result of the late Quaternary volcanism which is extinct or in dormant phase presently.

\section{CONCLUSIONS AND OUTLOOK}

Evolution of noble gas and water isotopes content along two transects of regional groundwater flow path in the Konya Closed Basin (KCB) of central Turkey revealed several facts. According to the ${ }^{3} \mathrm{He} /{ }^{4} \mathrm{He}$ versus $\mathrm{Ne} / \mathrm{He}$ plot of samples, crust and mantle appear to be the primary and secondary sources of dissolved $\mathrm{He}$ isotopes in groundwater. The ${ }^{3} \mathrm{He} /{ }^{4} \mathrm{He}$ versus $\mathrm{Ne} / \mathrm{He}$ plot appears to be a simple but effective tool for discriminating the dissolved gas sources in groundwater.

At the beginning of both transects where the flow domain is confined, He isotopes accumulate steadily in groundwater. Then, the intermediate recharge from the surface in the unconfined part of regional flow system disrupts the steady accumulation trend of $\mathrm{He}$ isotopes. Effect of intermediate recharge on regional groundwater is also indicated by the variations in specific conductance, temperature and water isotope and tritium signals.

The ${ }^{4} \mathrm{He}$ accumulation rates based on radiocarbon ages and ${ }^{4} \mathrm{He}$ concentrations along both transects between the mountain flank and Obruk Plateau are different in the eastern $\left(4.93 \times 10^{-11} \mathrm{~cm}^{3} \mathrm{STP} / \mathrm{g} \cdot y e a r\right)$ and western transects $\left(1.99 \times 10^{-10} \mathrm{~cm}^{3} \mathrm{STP} / \mathrm{g} \cdot \mathrm{year}\right)$. These values reveal that the ${ }^{4} \mathrm{He}$ accumulation rates may differ at least four times among parts of an aquifer 30 to $60 \mathrm{~km}$ apart from each other. Such variations may be due to spatially variable helium input and degassing to atmosphere both of which are affected by the geological/hydrogeological heterogeneities. It is understood that any groundwater age-dating by ${ }^{4} \mathrm{He}$ requires a firm understanding of the processes affecting the spatial rates of ${ }^{4} \mathrm{He}$ production and accumulation.

Finally, He isotope signal in groundwater seems to be a useful tool also to prove the mantle and/or crustal gas input into the carbonate aquifers where an on-going hypogenic karst development is suspected.

\section{ACKNOWLEDGEMENTS}

This study was funded by IAEA under the coordinated research project TUR-16283. Authors thank project coordinator Dr. Takuya Matsumoto (IAEA) and all members of other research groups in the coordinated project for their constructive discussions throughout the study. Support to field studies provided by Cave and Karst Research Group of the General Directorate of Mineral Research and Exploration, Turkey (MTA) is gratefully acknowledged. Constructive comments of Elise Fourré (Centre d'Etudes de Saclay) and three reviewers helped to improve the manuscript.

\section{REFERENCES}

Andrews J.N., 1985 - The isotopic composition of radiogenic He and its use to study groundwater movement in confined aquifer. Chemical Geology, 49: 339-351.

https://doi.org/10.1016/0009-2541(85)90166-4

Andrews J.N., 1992 - Mechanisms for noble gas dissolution by groundwaters. In: Isotopes of Noble Gases as Tracers in Environmental Studies, IAEA, p. 87-110.

Andrews J.N. \& Lee D.J., 1979 - Inert gases in groundwater from the Bunter Sandstone of England as indicators of age and paleoclimatic trends. Journal of Hydrology, 41: 233-252. https://doi.org/10.1016/0022-1694(79)90064-7

Andrews J.N., Giles I.S., Kay R.L.F. \& Lee D.J., 1982 Radioelemcnts, radiogenic helium, and age relationships for groundwaters from the granites at Stripa, Sweden. Geochimica et Cosmochimica Acta, 46: 1533-1543. https://doi.org/10.1016/0016-7037(82)90312-X

Andrews J.N., Goldbrunner J.E., Darling W.G., Hooker P.J., Wilson G.B., Youngman M.J., Eichinger L., Rauert W. \& Stichler W., 1985 - A radiochemical, hydrochemical and dissolved gas study of groundwaters in the Molasses basin of Upper Austria. Earth and Planetary Science Letters, 73: 317-332. https://doi.org/10.1016/0012-821X(85)90080-9

Balderer W. \& Lehmann B.E., $1989-{ }^{3} \mathrm{He} /{ }^{4} \mathrm{He}$-ratios as indicators of the origin of helium in groundwater; examples from the deep Nagra boreholes in northern Switzerland. In: D.L. Miles (Ed.), Water rock interaction. Balkema, Rotterdam, p. 45-47.

Ballentine C.J., O’Nions R.K., Oxburgh E.R., Horvath F. \& Deak J., 1991 - Rare gas constraints on hydrocarbon accumulation, crustal degassing and groundwater flow in the Pannonian Basin. Earth and Planetary Science Letters, 105 (1-3): 229-246.

https://doi.org/10.1016/0012-821X(91)90133-3

Bayari C.S., Ozyurt N.N. \& Kilani S., 2009a-Radiocarbon age distribution of groundwater in the Konya Closed Basin, central Anatolia, Turkey. Hydrogeology Journal, 17 (2): 347-365.

https://doi.org/10.1007/s10040-008-0358-2 
Bayari C.S., Pekkan E. \& Ozyurt N.N., 2009b-Obruks, as giant collapse dolines caused by hypogenic karstification in central Anatolia, Turkey: analysis of likely formation processes. Hydrogeology Journal, 17 (2): 327-345. https://doi.org/10.1007/s10040-008-0351-9

Bottomley D.J., Gascoyne M. \& Kamineni D.C., 1990 - The geochemistry, age, and origin of groundwater in a mafic pluton, East Bull Lake, Ontario, Canada. Geochimica et Cosmochimica Acta, 54 (4): 993-1008. https://doi.org/10.1016/0016-7037(90)90433-L

Clarke W.B., Jenkins W.B. \& Top Z., 1976-Determination of tritium by mass spectrometric measurements of ${ }^{3} \mathrm{He}$. The International Journal of Applied Radiation Isotopes, 27: 217-225.

https://doi.org/10.1016/0020-708X(76)90082-X

Farley K.A. \& Neroda E., 1998 - Noble gases in the Earth's mantle. Annual Review of Earth and Planetary Sciences, 26: 189-218. https://doi.org/10.1146/annurev.earth.26.1.189

Huai F.W., Ledoux E. \& de Marsily G., 1990 Regional modelling of groundwater flow and salt and environmental tracer transport in deep aquifers in the Paris Basin. Journal of Hydrology, 120: 341-358. https://doi.org/10.1016/0022-1694(90)90158-T

Gulec N., Hilton D.R. \& Mutlu H., 2002 - Helium isotope variations in Turkey: relationship to tectonics, volcanism and recent seismic activities. Chemical Geology, 187: 129-142.

https://doi.org/10.1016/S0009-2541(02)00015-3

Klimchouk A., 2015 - The karst paradigm: changes, trends and perspectives. Acta Carsologica, 44 (3): 289313. https://doi.org/10.3986/ac.v44i3.2996

Mamyrin B.A \& Tolstikhin I.N., $1984-$ Helium isotopes in nature. Elsevier, Amsterdam, 273 p.

Marty B., Torgersen T., Meynier V., O’Nions R.K. \& de Marsily G., 1993 - Helium isotope fluxes and groundwater ages in the Dogger Aquifer, Paris Basin. Water Resources Research, 29 (4): 1025-1035. https://doi.org/10.1029/93WR00007

Mazor E. \& Bosch A., 1991 - Dynamics of groundwater in deep basins ${ }^{4} \mathrm{He}$ dating, hydraulic discontinuities, and rates of drainage. International Conference on Groundwater in Large Sedimentary Basins. Australian Water Resources Council Conference Series, 20, p. 380-389.

Mazor E. \& Bosch A., 1992 - Helium as a semiquantitative tool for groundwater dating in the range of
104 to 108 years. In: Isotopes of noble gases as tracers in environmental studies, IAEA, p. 163-178.

Ozima M. \& Podosek F.A., 1983 - Noble gas geochemistry. Cambridge University Press, 367 p.

Ozyurt N.N. \& Bayari C.S., 2014 - Helium isotopes as indicator of current hypogenic karst development in Taurids karst region, Turkey. In: A. Klimchouk, I. Sasowsky, J. Mylroie, S.A. Engel \& A.S. Engel (Eds.), Hypogene cave morphologies. Karst Waters Institute Special Publication, 18, p. 77-81.

Ozyurt N.N. \& Bayari C.S., 2015 - Evolution of noble gas isotopes along the regional groundwater flow path: Konya Closed Basin, Turkey. IAEA Symposium, Vienna.

Rozanski K., 1992 - Summary of consultants meeting on isotopes of noble gases as tracers in environmental studies. In: Isotopes of moble gases as tracers in environmental studies, IAEA, $305 \mathrm{p}$.

Solomon D.K., $2000-{ }^{4}$ He in groundwater. In: P.Cook \& A.L. Herczeg (Eds.), Environmental tracers in subsurface hydrology. Springer, Berlin, p. 425-439. https://doi.org/10.1007/978-1-4615-4557-6 14

Stute M., Sonntag C., Deak J. \& Schlosser P., 1992 Helium in deep circulating groundwater in the Great Hungarian Plain: flow dynamics and crustal and mantle helium fluxes. Geochimica et Cosmochimica Acta, 56: 2051-2067.

https://doi.org/10.1016/0016-7037(92)90329-H

Torgersen T., 1980 - Controls on pore-fluid concentration of ${ }^{4} \mathrm{He}$ and ${ }^{222} \mathrm{Rn}$ and the calculation of ${ }^{4} \mathrm{He} /{ }^{222} \mathrm{Rn}$ ages. Journal of Geochemical Exploration, 13: 57-75. https://doi.org/10.1016/0375-6742(80)90021-7

Torgersen T. \& Clarke W.B., 1985 - Helium accumulation in groundwater, I: An evaluation of sources and the continental flux of crustal ${ }^{4} \mathrm{He}$ in the Great Artesian Basin, Australia. Geochemica et Cosmochimica Acta, 49: 1211-1218. https://doi.org/10.1016/0016-7037(85)90011-0

Torgersen T. \& Ivey G.N., 1985 - Helium accumulation in groundwater. II: A model for the accumulation of the crustal ${ }^{4} \mathrm{He}$ degassing flux. Geochimica et Cosmochimica Acta, 49: 2445-2452. https://doi.org/10.1016/0016-7037(85)90244-3

UNESCO, 1972 - Glossary and multilingual equivalents of karst terms. UNESCO, Paris, 77 p.

Walthan T., 2015 - Large collapse sinkholes, old and new, in the Obruk Plateau, Turkey. Cave and Karst Science, 42 (3): 125-130.

http:/ / bcra.org.uk/pub/candks/index.html?j=126 